$16 \%$ pred.; $\mathrm{SpO}_{2}(\%)$ rest: $\left.95 \pm 2\right)$ were equipped with a portable Jaeger Oxycon Mobile ${ }^{\circledR}$ metabolic system and followed an audio signal for stepping up and down a single $20 \mathrm{~cm}$ step for 3 minutes. Borg dyspnea scores were obtained at the end each stepping bout. A 10-min rest was given between each stepping bout.

Results: O f the 43 patients, $80 \%$ completed stages 1 and 2, 74 and $37 \%$ stages 3 and 4 while no patient of MRC class 4 or $5(n=8)$ completed stage 1 . Breathing frequency (breaths. $\mathrm{min}^{-1}$ ) spanned from $26.5 \pm 4.1$ to $39.0 \pm 6.4$ but VT (L) remained unchanged (1.4 0.3 vs. 1.5 \pm 0.4 ) from stage 1 to 4 while Borg scores were $3 \pm 1,4 \pm 1,5 \pm 2,6 \pm 3$ respectively and $\mathrm{SpO}_{2}(\%)$ were $92 \pm 5,91 \pm 4,91 \pm 4$ and $90 \pm 4$.

Conclusions: Preliminary findings indicate that a 3min constant rate step test may present a feasible alternative to laboratory testing to assess exertional dyspnea in moderately severe COPD. In this population, a stepping rate of $26 \mathrm{steps} \cdot \mathrm{min}^{-1}$ could be sustained by the majority of patients while producing a level of dyspnea potentially amenable to therapy.

This study was supported by an unrestricted grant from Boehringer-Ingelheim/Pfizer in the Primary Care Setting in Patients with COPD.

\section{Quality Assurance and the Service Domain in Car- diac and Pulmonary Rehabilitation}

Hoberty, PD, Respiratory Therapy Division, School of Allied Medical Professions, The Ohio State

University, Columbus, Ohio, USA

Background: Little is known about the quality assurance methods used on cardiac and pulmonary rehabilitation. Also, little is known as to what extent the service domain is evaluated and what methods are employed. Knowledge of what is being done in these regards may facilitate programs effectively and efficiently measuring these outcomes.

Methods: A survey was mailed to $1 / 3$ of the programs (approximately 400) in both cardiac and pulmonary rehabilitation listed in the AACVPR Program Guide 2004. The survey included questions that would add to the knowledge of what is the extent of practice and methods. 12 components of quality assurance were listed and 14 components of patient satisfaction were assessed as to the frequency of use. It was pilot tested and revised.

Results: The usable return rate was $48 \%$. Program directors of both types of programs claimed daily formal assessment of most of the 12 areas listed for quality assurance. The three highest being assessment of adverse events, safety, and patient satisfaction. The three lowest use was in the area of efficiency, timeliness and continuity of care. The service domain was assessed as comparable to the other three: health, clinical and behavioral domains. Patient satisfaction was the most commonly assessed component of the service domain with more than $90 \%$ of both types of programs measuring program effectiveness, overall program quality and friendliness of the staff. Managers most commonly cited the acquisition of new equipment as the endpoint as the end results of patient satisfaction assessment.

Conclusions: There was surprising similarity in results from programs in cardiac and pulmonary rehabilitation. Quality assurance is very frequently assessed in both. Concern for adverse events, safety, and patient satisfaction predominate.

\section{Use of Preparatory Stability Exercises with Chronic Obstructive Pulmonary Disease Patients (COPD) to Prevent Iatrogenic Injuries during Rehabilitation}

Holly, Janet T; Lavallee, Lyne G, The Rehabilitation Centre of the Ottawa Hospital, Ottawa, Ontario, Canada

Background: The increasing incidence of injuries sustained by clients during pulmonary rehabilitation, created a need to develop a prevention strategy. A prepulmonary rehabilitation stability exercise class was created based on best practice principles from the orthopaedic literature. It has been discussed in the literature that patients who have COPD have poor stability strategies based on the dominance of the need to drive the respiratory system. If successful, it was felt that this program would decrease the incidence of in- 
jury, decrease length stay and help to optimize outcomes.

Methods: Six months of data recording the incidence and severity of injuries from participants in the pulmonary rehabilitation program was collected in order to obtain comparative statistics and demonstrate the need for this program. A literature review was performed to determine the risk of injury in this population. In a 6 month period, $17 \%$ of COPD clients admitted to the Rehabilitation Centre for pulmonary rehabilitation have had musculoskeletal issues that proved a significant enough barrier to rehabilitation to require treatment or pulmonary rehabilitation modification. Wait list clients for pulmonary rehabilitation were assessed using the PSFS, NPRS, the 6-minute walk test, the Active Straight Leg Raise, Sitting Arm Lift and the non-stop walk test. Clients participated in six one-hour group exercise sessions. The exercises included neck stabilizers, pelvic floor muscles, trunk stabilizers, and scapular stabilizers derived from published literature.

Results: Clients were re-evaluated using the same outcome tools as well as noting any injuries sustained and capacity to participate. This data was compared with historical data.

Conclusion: The data will help With patient selection for participation in the exercise class as well as refinement of the outcome tools and exercise protocol. This project illustrates the opportunities that exist to share and transfer knowledge from one area of physiotherapy expertise to another to meet the needs of practice. It is essential that this integrated approach to treatment be fostered by clinicians, educators and researchers alike to improve the overall outcomes for the clients.

\section{Times to Treatment in Patients Undergoing Pri- mary Percutaneous Coronary Intervention at La- val Hospital}

Lachance Philippe, Garceau Patrick, Déry JeanPierre, Institut universitaire de cardiologie et de pneumologie, Hôpital Laval, Québec, Québec, Canada

Background:Current guidelines for ST-elevation myocardial infarction (STEMI) recommend perform- ing primary percutaneous coronary intervention (PCI) within $90 \mathrm{~min}$ of hospital arrival. However, recent data suggest that, in a real-world setting, median doorto-balloon (DTB) time is closer to 180 minfor transferred patients with no more than $4 \%$ of patients treated within $90 \mathrm{~min}$. We conducted this retrospective observational study to assess time to treatment in patients undergoing primary PCI at Quebec Heart and Lung Institute (QHLI).

Methods: Consecutive lytic-eligible patients undergoing primary PCI at QHLI for STEMI between April 2004 and March 2004 were included in the present analysis. The primary evaluation was DTB time measured from arrival at the first hospital to first balloon inflation. Clinical outcomes consisted of inhospital death, reinfarction and bleeding. DTB times and hospital outcomes of patients transferred from referring hospitals were compared to patients presenting directly to QHLI.

Results: During the study period, 203 lytic-eligible patients were treated with primary PCI and included in the present analysis. Sixty-nine patients presented directly to QHLI and 134 were transferred from other Quebec City hospitals. The median DTB time was 114 min in transferred patients, compared with $87 \mathrm{~min}$ for patients presenting directly to QHLI $[P<0.001]$. DTB time was $<90$ min in $24 \%$ of the transferred population compared with $55 \%$ for patients presenting directly to QHLI $[P<0.001]$. Clinical outcomes did not differ among the two groups.

Conclusion: DTB time of $<90$ min can be achieve in the majority of patients who present directly to a primary PCI center whereas there is room for improvement when patients are transferred from a community hospital. This study highlights the need for standardized transfer protocol to improve DTB time in patients transferred for primary PCI from community hospitals. 